\title{
2-Alkoxycarbonylcycloimmonium Ylides, Efficient 1,4-Dipole Equivalents in the Synthesis of New Conjugated Betaines.
}

\author{
Ana M. Cuadro, Jesús Valenciano, Juan J. Vaquero, José L. García Navío and Julio Alvarez-Builla. \\ Departamento de Química Orgánica, Universidad de Alcalá. 28871 Alcalá de Henares. Madrid. Spain.
}

(Received in UK 25 November 1992)

Key words: azinium ylides; pyrido[1,2-a]pyrazine; pyrido[2,1-f][1,2,4]triazine; synthesis; mesomeric betaines.

Abstract: Several heterocyclic mesomeric betaines containing the bicyclic systems pyrido[1,2-a]pyrazine and pyrido $[2,1-f][1,2,4]$ triazine have been prepared by reaction of 2 -alkoxycarbonyl pyridinium $\mathrm{N}$-ylides with phenyl isocyanate and isothiocyanate.

2-Alkoxycarbonylpyridinium ylides 2 (Scheme 1) are interesting species as they should eventually behave as 1,4-dipoles able to produce, by reaction with the corresponding dipolarophiles, derivatives 3 . Such a strategy has not been described in the literature, apart from a reaction of 2-carbonyl- $\mathrm{N}$-iminopyridinium ylides with amides or nitriles, producing pyrido[2,1-f]-as-triazinium-1 and 3-olates. ${ }^{1-3}$ Related heterobetaines have been obtained either from pyridinium precursors ${ }^{4,5}$ or from 2 -functionalized pyrilium salts. ${ }^{6-9}$

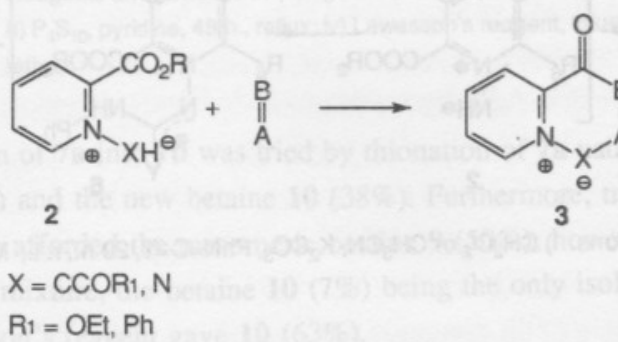

\section{Scheme 1}

In this paper we wish to report the synthetic utility of 2-alkoxycarbonyl pyridinium $\mathrm{N}$-ylides $\mathbf{2}$ as intermediates for the synthesis of mesomeric betaines 5 and 7 (Scheme 2 and 3) both classified as conjugated and isoconjugate with even alternant hydrocarbon dianions according to Ollis. ${ }^{10}$

$\mathrm{N}$-ylides $\mathbf{2} \mathbf{a}$ and $\mathbf{2 b}$ are readily generated from 2 -ethoxycarbonyl pyridinium salts $(\mathbf{1 a}, \mathbf{1} \mathbf{b})$, and reacted with phenyl isocyanate affording the corresponding pyrido[1,2-a]pyrazinium-3-olates $5(Y=0)$ in good yield (see table 1), together with detectable amounts of compounds $4(Y=0)$. Similarly, $\mathrm{N}$-imino compounds $1 \mathrm{c}$-f gave the corresponding pyrido[2,1-f][1,2,4] triazinium-3-olates and their benzologues $7(Y=0)$ in excellent yield (see table 2). 


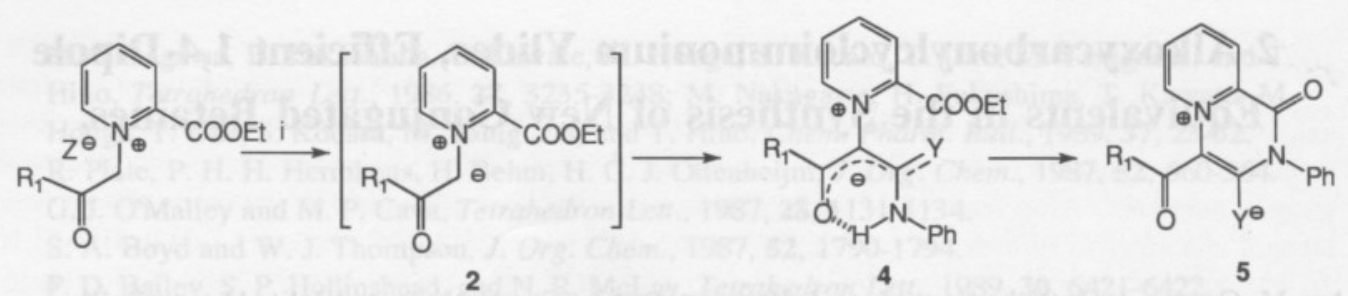

1a, $R_{1}=O E t, Z=B r$

1b, $\mathrm{R}_{1}=\mathrm{Ph}, \mathrm{Z}=\mathrm{Br}$

Scheme 2. Reagents and condifions: i) $\mathrm{CH}_{2} \mathrm{Cl}_{2}$ or $\mathrm{CH}_{3} \mathrm{CN}, \mathrm{K}_{2} \mathrm{CO}_{3}, \mathrm{PhN}=\mathrm{C}=\mathrm{Y}, 20$ h., r.t.

Table 1. Betaines $\mathbf{4}$ and $\mathbf{5}$ prepared.

\section{Starting}

Comp. Material $\mathbf{Y}$

$\begin{array}{ll}4,5 a & 1 a \\ 4,5 b & 1 a \\ 4,5 c & 1 b \\ 4,5 d & 1 b\end{array}$

$\mathrm{O}$

S

$\mathrm{O}$

S

\section{$\mathbf{R}_{1}$}

OEt

$\mathrm{OEt}$

$\mathrm{Ph}$

$\mathrm{Ph}$

\section{Yield \%}

45

$\begin{array}{ll}3 & 70 \\ 30 & 63 \\ \text { traces } & 68 \\ 31 & 66\end{array}$

Compounds 7 ( $\mathrm{Y}=\mathrm{S}$ ) were directly obtained when phenyl isothiocyanate was added to a suspension of the salts $1 \mathrm{c}-\mathrm{f}$ in dichloromethane and potassium carbonate. However, following the above procedure, the salts 1a and $\mathbf{1 b}$ afforded compounds $4(\mathrm{Y}=\mathrm{S})$ which underwent easy cyclization to $5(\mathrm{Y}=\mathrm{S})$ in the presence of triethylamine.<smiles>[R]c1c([R])c([R])[n+](N)c(C(=O)O)c1[2H]</smiles>

1c-f, $Z=$ MSTS

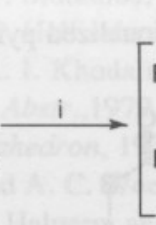

$\left[\begin{array}{l}R_{5} \\ R_{6}\end{array}\right.$<smiles>[R6]c1c([R])c([2H])[n+]([N+](=O)[O-])c(C(=O)O)c1[R]</smiles>

2

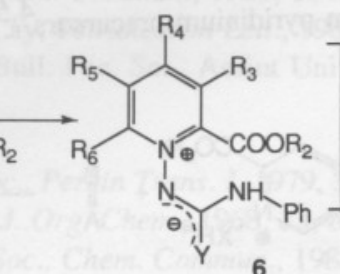

6

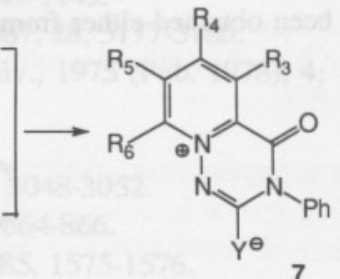

7

Scheme 3. Reagents and conditions: i) $\mathrm{CH}_{2} \mathrm{Cl}_{2}$ or $\mathrm{CH}_{3} \mathrm{CN}, \mathrm{K}_{2} \mathrm{CO}_{3}, \mathrm{PhN}=\mathrm{C}=\mathrm{Y}, 20$ h., r. t; $\mathrm{MSTS}=$ mesytilenesulphonate.

All new compounds gave satisfactory spectroscopic (IR, MS, ${ }^{1} \mathrm{H} /{ }^{13} \mathrm{C}-\mathrm{NMR}$ ) and analytical data. IR spectra of compounds 7 were highly characteristic. As an example, 7a shows a strong $\mathrm{C}=\mathrm{O}$ stretching band at $1700 \mathrm{~cm}^{-1}$ in addition to the band at $1635 \mathrm{~cm}^{-1}$ attributable to the 3-olate group. Alternatively, $7 \mathrm{~b}$ only shows a strong band at $1701 \mathrm{~cm}^{-1}$.

Several transformations were tested, as indicated in Scheme 4, allowing the preparation of new derivatives. Conversion of $\mathbf{7 b}$ into $\mathbf{7 a}$ was attempted via $\mathbf{8}$, obtained by S-methylation of $\mathbf{7 b}$, but $\mathbf{8}$, when submitted to basic hydrolysis gave decomposition products. 
Table 2. Betaines 7 prepared.

\section{Starting}

$\begin{array}{llllllllll}\text { Comp. } & \text { Material } & \mathbf{Y} & \mathbf{R}_{2} & \mathbf{R}_{\mathbf{3}} & \mathbf{R}_{\mathbf{4}} & \mathbf{R}_{\mathbf{5}} & \mathbf{R}_{\mathbf{6}} & \text { Yield \% }\end{array}$

$\begin{array}{lllllllll}\text { 7a } & \text { 1c } & \text { O } & \text { Et } & \text { H } & \text { H } & \text { H } & \text { H } & 74 \\ \text { 7b } & \text { 1c } & \text { S } & \text { Et } & \text { H } & \text { H } & \text { H } & \text { H } & 70 \\ \text { 7c } & \text { 1d } & \text { O } & \text { Et } & \text { H } & \text { H } & (\mathrm{CH}=\mathrm{CH})_{2} & 66 \\ \text { 7d } & \text { 1d } & \text { S } & \text { Et } & \text { H } & \text { H } & (\mathrm{CH}=\mathrm{CH})_{2} & 53 \\ \text { 7e } & \text { 1e } & \text { O } & \text { Et } & (\mathrm{CH}=\mathrm{CH})_{2} & \mathrm{H} & \mathrm{H} & 80 \\ \text { 7f } & \text { 1e } & \text { S } & \text { Et } & (\mathrm{CH}=\mathrm{CH})_{2} & \mathrm{H} & \mathrm{H} & 61 \\ \text { 7g } & \text { 1f } & \text { O } & \mathrm{Me} & \mathrm{H} & (\mathrm{CH}=\mathrm{CH})_{2} & \mathrm{H} & 60 \\ \text { 7h } & \text { 1f } & \text { S } & \mathrm{Me} & \mathrm{H} & (\mathrm{CH}=\mathrm{CH})_{2} & \mathrm{H} & 45\end{array}$
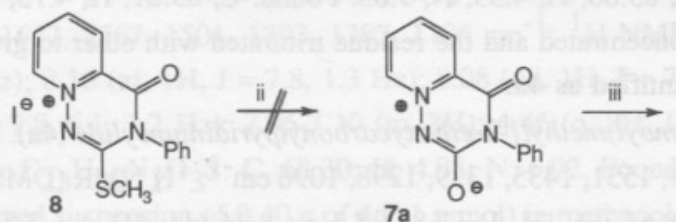

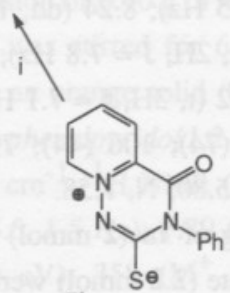

$7 \mathrm{~b}$
$7 a$

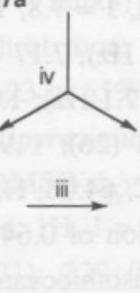

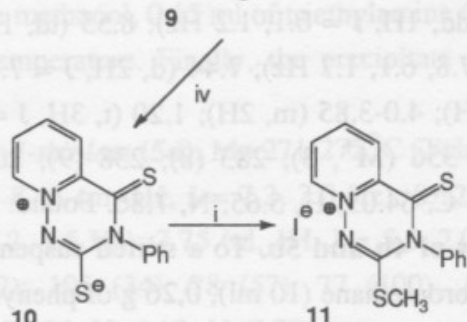

10<smiles></smiles>

Scheme 4. Reagents and conditions: i) $\mathrm{CH}_{3}$ l, $\mathrm{AcOEt}$, r. t.; ii) $\mathrm{NaOH}$ (aq., 50\%), r. t.;

iii) $\mathrm{P}_{4} \mathrm{~S}_{10}$. pyridine, $48 \mathrm{~h}$., reflux; iv) Lawesson's reagent, toluene, $72 \mathrm{~h}$. reflux.

Alternative conversion of $\mathbf{7 a}$ into $\mathbf{7 b}$ was tried by thionation of 7a using Lawesson's reagent in boiling toluene, affording $\mathbf{7 b}(20 \%)$ and the new betaine 10 (38\%). Furthermore, treatment of $7 \mathrm{a}$ with phosphorus pentasulfide in dry pyridine afforded the mesomeric betaine $9(50 \%)$; however, reaction of $7 \mathbf{b}$ under similar conditions gave a complex mixture, the betaine $10(7 \%)$ being the only isolated product. On the other hand, treatment of 9 with Lawesson's reagent gave $10(63 \%)$.

In summary, a simple and useful preparation of new betaines 5 and 7 have been achieved and alternative methods have surfaced for the synthesis of betaines 10. Further experiments are in progress to extend this methodology to other heterocyclic systems.

\section{EXPERIMENTAL}

Melting points were determined on an Electrothermal IA6304 and are uncorrected. IR spectra were recorded on Perkin-Elmer 700 or 1310 spectrophotometers using $\mathrm{KBr}$ pellets. ${ }^{1} \mathrm{H}-\mathrm{y}{ }^{13} \mathrm{C}-\mathrm{NMR}$ spectra were recorded on a Varian Unity 300 instrument at 300 and $75.429 \mathrm{MHz}$ respectively. Mass spectra were 
determined on a Hewlett-Packard 5988A $(70 \mathrm{eV})$ spectrometer. Satisfactory microanalyses were obtained for all new compounds described, within $0.4 \%$ error.

The starting heterocyclic precursors were obtained using previously described methods. ${ }^{4,11,12}$

Synthesis of $4 \mathbf{a}$ and $5 \mathbf{a}$. To a stirred suspension of $0.63 \mathrm{~g}$ of the pyridinium salt $1 \mathrm{a}(2 \mathrm{mmol})$ and 1.10 $\mathrm{g}$ of $\mathrm{K}_{2} \mathrm{CO}_{3}(8 \mathrm{mmol})$ in dry acetonitrile $(10 \mathrm{ml}), 0.24 \mathrm{ml}$ of phenyl isocyanate $(2.2 \mathrm{mmol})$ were added, and the reaction mixture was stirred at room temperature for $20 \mathrm{~h}$. Then, the inorganic residue was filtered off and the liquid was concentrated to dryness. The resulting residue was triturated with $10 \mathrm{ml}$ of ethyl acetate, yielding $5 \mathrm{a}$ as a yellow crystalline solid $(0.43 \mathrm{~g}, 70 \%)$

4-Ethoxycarbonyl-1-oxo-2-phenylpyrido[1,2-a]pyraziniun-3-olate (5a). $\mathrm{Mp} 249-250^{\circ} \mathrm{C}(\mathrm{EtOH}) . \mathrm{IR}(\mathrm{KBr})$ : $1688,1641,1453,1323,1206 \mathrm{~cm}^{-1} ;{ }^{1} \mathrm{H}$ NMR (DMSO-d 6$) \delta 9.70(\mathrm{~d}, 1 \mathrm{H}, \mathrm{J}=6.9 \mathrm{~Hz}) ; 8.40(\mathrm{dd}, 1 \mathrm{H}, \mathrm{J}=7.8$, $2.0 \mathrm{~Hz}) ; 7.90(\mathrm{td}, 1 \mathrm{H}, \mathrm{J}=6.9,2.0 \mathrm{~Hz}) ; 7.80(\mathrm{td}, 1 \mathrm{H}, \mathrm{J}=7.8,1.2 \mathrm{~Hz}) ; 7.51-7.20(\mathrm{~m}, 5 \mathrm{H}) ; 4.19$ (q, 2H, J = 7.1 $\mathrm{Hz}) ; 1.21(\mathrm{t}, 3 \mathrm{H}, \mathrm{J}=7.1 \mathrm{~Hz})$ ppm; MS $(70 \mathrm{eV}) \mathrm{m} / \mathrm{e}$ (rel intensity): $310\left(\mathrm{M}^{+}, 24\right) ; 238$ (70); $106(23) ; 78$ (100). Anal. calcd. for $\mathrm{C}_{17} \mathrm{H}_{14} \mathrm{~N}_{2} \mathrm{O}_{4}$ : C, 65.80; H, 4.55; N, 9.03. Found: C, 65.81; H, 4.75; N, 9.20.

The remaining ethyl acetate was concentrated and the residue triturated with ether to give $20 \mathrm{mg}$ ( $3 \%$ ) of a red powder whose structure was identified as $\mathbf{4 a}$.

1 -[(Ethoxycarbonyl-N-phenylcarbamoyl)methyl]-2-ethoxycarbonylpyridinium ylide (4a). Mp $110-111^{\circ} \mathrm{C}$ (Et $\left.{ }_{2} \mathrm{O}\right)$. IR (KBr): 1745, 1638, 1611, 1579, 1531, 1435, 1339, 1298, $1096 \mathrm{~cm}^{-1} ;{ }^{1} \mathrm{H}$ NMR (DMSO-d 6 ) $\delta 10.57$ $(\mathrm{s}, 1 \mathrm{H}) ; 8.91(\mathrm{dd}, 1 \mathrm{H}, \mathrm{J}=6.1,1.2 \mathrm{~Hz}) ; 8.55(\mathrm{td}, 1 \mathrm{H}, \mathrm{J}=7.8,1,5 \mathrm{~Hz}) ; 8.24(\mathrm{dd}, 1 \mathrm{H}, \mathrm{J}=7.8,1.7 \mathrm{~Hz}) ; 8.09$ (ddd, $1 \mathrm{H}, \mathrm{J}=7.8,6.1,1.7 \mathrm{~Hz}) ; 7.44(\mathrm{~d}, 2 \mathrm{H}, \mathrm{J}=7.8 \mathrm{~Hz}) ; 7.17(\mathrm{t}, 2 \mathrm{H}, \mathrm{J}=7.8 \mathrm{~Hz}) ; 6.85(\mathrm{t}, 1 \mathrm{H}, \mathrm{J}=7.2 \mathrm{~Hz}$ ); 4.35-.15 (m, 2H); 4.0-3.85 (m, 2H); $1.20(\mathrm{t}, 3 \mathrm{H}, \mathrm{J}=7.1 \mathrm{~Hz}) ; 1.02(\mathrm{t}, 3 \mathrm{H}, \mathrm{J}=7.1 \mathrm{~Hz}) \mathrm{ppm}$; MS (70 eV) m/e (rel intensity): $356\left(\mathrm{M}^{+}, 3\right) ; 283$ (8); 238 (9); 208 (26); 119 (74); 106 (44); 79 (100). Anal. calcd. for $\mathrm{C}_{19} \mathrm{H}_{20} \mathrm{~N}_{2} \mathrm{O}_{5}$ : C, 64.05; H, 5.65; N, 7.86. Found: C, 64.06; H, 5.80; N, 7.58.

Synthesis of $4 \mathrm{~b}$ and $5 \mathrm{~b}$. To a stirred suspension of $0.64 \mathrm{~g}$ of $1 \mathrm{a}(2 \mathrm{mmol})$ and $0.55 \mathrm{~g}^{\circ} \mathrm{K}_{2} \mathrm{CO}_{3}(4$ $\mathrm{mmol})$ in dichloromethane $(10 \mathrm{ml}), 0,26 \mathrm{~g}$ of phenyl isothiocyanate $(2.2 \mathrm{mmol})$ were added. The mixture was stirred for $20 \mathrm{~h}$ at room temperature. Then, the inorganic solid was separated by filtration and the liquids were concentrated to dryness. The residue was purified by column chromatography (silica gel 60 A, 230-400 mesh; ethyl acetate) yielding $\mathbf{4 b}$ as a red solid $(0.21 \mathrm{~g}, 30 \%)$.

I-I(Ethoxycarbonyl-N-phenylthiocarbamoyl)methyl]-2-ethoxycarbonylpyridinium ylide (4b). Mp 100$101^{\circ} \mathrm{C}\left(\mathrm{Et}_{2} \mathrm{O}\right) . \mathrm{IR}(\mathrm{KBr}): 1741,1587,1400,1373,1346 \mathrm{~cm}^{-1} ;{ }^{1} \mathrm{H} \mathrm{NMR}\left(\mathrm{CDCl}_{3}\right) \delta 11.84(\mathrm{~s}, 1 \mathrm{H}) ; 8.68(\mathrm{dd}$, $1 \mathrm{H}, \mathrm{J}=6.1 \mathrm{~Hz}$ ); 8.32 (td, $1 \mathrm{H}, \mathrm{J}=7.8,1.5 \mathrm{~Hz}) ; 8.13$ (dd, $1 \mathrm{H}, \mathrm{J}=7.8,1.7 \mathrm{~Hz}) ; 7.84$ (ddd, $1 \mathrm{H}, \mathrm{J}=7.8,6.1$, $1.7 \mathrm{~Hz}) ; 7.73$ (d, 2H, J = 8.5 Hz); 7.29 (t, 2H, J = 7.6 Hz); $7.06(\mathrm{t}, 1 \mathrm{H}, \mathrm{J}=7.3 \mathrm{~Hz}) ; 4.5-4.3(\mathrm{~m}, 2 \mathrm{H}) ; 4.15-$ $3.95(\mathrm{~m}, 2 \mathrm{H}) ; 1.35(\mathrm{t}, 3 \mathrm{H}, \mathrm{J}=7.1 \mathrm{~Hz}) ; 1.09(\mathrm{t}, 3 \mathrm{H}, \mathrm{J}=7.1 \mathrm{~Hz}) \mathrm{ppm}$. Anal. calcd. for $\mathrm{C}_{19} \mathrm{H}_{20} \mathrm{~N}_{2} \mathrm{O}_{4} \mathrm{~S}: \mathrm{C}$, 61.27 ; H, 5.41; N, 7.52. Found: C, 60.98; H, 5.19; N, 7.28.

To a stirred solution of $0.38 \mathrm{~g}$ of $\mathbf{4 b}(1 \mathrm{mmol})$ in methanol $(10 \mathrm{ml}), 0.15 \mathrm{ml}$ of triethylamine $(1.1 \mathrm{mmol})$ were added. The mixture was stirred for $6 \mathrm{~h}$ at room temperature. Then, the precipitate was isolated by filtration, yielding $\mathbf{5 b}$ as a red crystalline solid $(0.20 \mathrm{~g}, 62 \%)$

4-Ethoxycarbonyl-1-oxo-2-phenylpyrido[1,2-a]pyrazinium-3-thiolate (5b). Mp 204-205 ${ }^{\circ} \mathrm{C}$ (EtOH). IR (KBr) $1709,1675,1451,1413,1371,1216 \mathrm{~cm}^{-1}$; ${ }^{1} \mathrm{H}$ NMR (DMSO-d 6 ) $\delta 8.33$ (dd, $1 \mathrm{H}, \mathrm{J}=8.0 \mathrm{~Hz}$ ); 8.16 (d, $1 \mathrm{H}, \mathrm{J}=6.6 \mathrm{~Hz}) ; 7.86(\mathrm{td}, \mathrm{J}=7.3,6.6,1.7 \mathrm{~Hz}) ; 7.78(\mathrm{t}, 1 \mathrm{H}, \mathrm{J}=8.0,7.3 \mathrm{~Hz}) ; 7.50-7.15(\mathrm{~m}, 5 \mathrm{H}) ; 4.34(\mathrm{q}, 2 \mathrm{H}$, $\mathrm{J}=7.2 \mathrm{~Hz}) ; 1.29(\mathrm{t}, 3 \mathrm{H}, \mathrm{J}=7.2 \mathrm{~Hz}) \mathrm{ppm}$; MS $(70 \mathrm{eV}) \mathrm{m} / \mathrm{e}$ (rel intensity) $326\left(\mathrm{M}^{+}, 28\right) ; 297$ (25); $253(52)$; 106 (20); 78 (100). Anal. calcd. for $\mathrm{C}_{21} \mathrm{H}_{14} \mathrm{~N}_{2} \mathrm{O}_{3} \mathrm{~S}$ : C, 62.56; H, 4.32; N, 8.58. Found: C. 62.28; H, 4.14; $\mathrm{N}, 8.24$.

4-Benzoyl-1-oxo-2-phenylpyrido[1,2-a]pyrazinium-3-olate (5c). To a suspension of $0.70 \mathrm{~g}$ of the 
pyridinium salt $1 \mathrm{~b}(2 \mathrm{mmol})$ and $1.10 \mathrm{~g}$ of $\mathrm{K}_{2} \mathrm{CO}_{3}(8 \mathrm{mmol})$ in dry acetonitrile, $0.24 \mathrm{ml}$ of phenyl isocyanate ( $2.2 \mathrm{mmol}$ ) were added. The mixture was stirred for $20 \mathrm{~h}$ at room temperature. Then, the precipitate was isolated by filtration and washed with distilled water until neutral, yielding the betaine $\mathbf{5} \mathbf{c}$ which crystallised from ethanol affording yellow crystals $(0.47 \mathrm{~g}, 70 \%)$. Mp $264-265^{\circ} \mathrm{C}(\mathrm{EtOH})$; $\mathrm{IR}(\mathrm{KBr}) 1683,1632,1449$, $1334,1197 \mathrm{~cm}^{-1}$; ${ }^{1} \mathrm{H}$ NMR (DMSO-d $) ~ \delta 10.18(\mathrm{~m}, 1 \mathrm{H}, \mathrm{J}=6.0,1.8 \mathrm{~Hz}) ; 8.51(\mathrm{~m}, 1 \mathrm{H}, \mathrm{J}=7.2,2.9 \mathrm{~Hz}$ ); 8.02-7.92 (m, 2H); 7.60-7.19 (m, 10H) ppm; MS (70 eV) m/e (rel intensity): $342\left(\mathrm{M}^{+}, 80\right) ; 265(16) ; 195$ (71); 105 (100). Anal. calcd. for $\mathrm{C}_{21} \mathrm{H}_{14} \mathrm{~N}_{2} \mathrm{O}_{3}$ : C, 73.67; H, 4.12; N, 8.18. Found: $\mathrm{C}, 74.01 ; \mathrm{H}, 4.41 ; \mathrm{N}, 8.04$.

Synthesis of $4 \mathrm{~d}$ and $5 \mathrm{~d}$. To a stirred suspension of $0.70 \mathrm{~g}$ of $1 \mathrm{~b}(2 \mathrm{mmol})$ and $0.55 \mathrm{~g}^{\circ} \mathrm{K}_{2} \mathrm{CO}_{3}(4$ $\mathrm{mmol})$ in dichloromethane $(10 \mathrm{ml}), 0.26 \mathrm{ml}$ of phenyl isothiocyanate $(2.2 \mathrm{mmol})$ were added. The mixture was stirred for $20 \mathrm{~h}$ at room temperature. Then, the inorganic residue was separated by filtration and the liquids were concentrated to dryness. The residue was purified by column chromatography (silica gel $60 \mathrm{~A}, 230-400$ mesh; ethyl acetate) yielding the betaine $\mathbf{4 d}$ as a red solid $(0.25 \mathrm{~g}, 31 \%)$.

1-I(Benzoyl-N-phenylthiocarbamoyl)methyl]-2-ethoxycarbonylpyridinium ylide (4d). Mp 134-135 ${ }^{\circ} \mathrm{C}$. IR $(\mathrm{KBr}): 1734,1627,1567,1501,1393,1307,1195 \mathrm{~cm}^{-1} ;{ }^{1} \mathrm{H}$ NMR $\left(\mathrm{CDCl}_{3}\right) \delta 13.62(\mathrm{~s}, 1 \mathrm{H}) ; 8.42(\mathrm{dd}, 1 \mathrm{H}$, $\mathrm{J}=6.1,1.3 \mathrm{~Hz}) ; 8.16(\mathrm{td}, 1 \mathrm{H}, \mathrm{J}=7.8,1.3 \mathrm{~Hz}) ; 8.08(\mathrm{dd}, 1 \mathrm{H}, \mathrm{J}=7.8,1.7 \mathrm{~Hz}) ; 7.83(\mathrm{~d}, 2 \mathrm{H}, \mathrm{J}=7.4 \mathrm{~Hz}) ; 7.56$ (ddd, $1 \mathrm{H}, \mathrm{J}=7.8,6.1,1.7 \mathrm{~Hz}) ; 7.36-7.10(\mathrm{~m}, 8 \mathrm{H}) ; 4.46(\mathrm{q}, 2 \mathrm{H}, \mathrm{J}=7.1 \mathrm{~Hz}) ; 1.41(\mathrm{t}, 3 \mathrm{H}, \mathrm{J}=7.1 \mathrm{~Hz}) \mathrm{ppm}$. Anal. calc. for $\mathrm{C}_{23} \mathrm{H}_{20} \mathrm{~N}_{2} \mathrm{O}_{3} \mathrm{~S}$ : C, 68.30; H, 4.98; N, 6.92. Found: C, 68.10; H, 4.80; N, 6.71.

To a stirred suspension of $0.40 \mathrm{~g}$ of $\mathbf{4 d}(1 \mathrm{mmol})$ in methanol, $0.15 \mathrm{ml}$ of triethylamine $(1.1 \mathrm{mmol})$ were added, and the mixture was stirred for $6 \mathrm{~h}$ at room temperature. Finally, the precipitate was isolated by filtration, yielding $5 \mathrm{~d}$ as an orange solid $(0.23 \mathrm{~g}, 65 \%)$.

4-Benzoyl-1-oxo-2-phenylpyrido[1,2-a]pyrazinium-3-thiolate (5d). $\mathrm{Mp} 271-272^{\circ} \mathrm{C}$ (Toluene). $\mathrm{IR}(\mathrm{KBr})$ : $1666,1450,1419,1219 \mathrm{~cm}^{-1} ;{ }^{1} \mathrm{H}$ NMR (DMSO-d 6 ) $\delta 8.43(\mathrm{~m}, 1 \mathrm{H}, \mathrm{J}=7.2,2.0 \mathrm{~Hz}$ ); $8.12(\mathrm{~d}, 2 \mathrm{H}, \mathrm{J}=7.1$ $\mathrm{Hz}) ; 7.96(\mathrm{dd}, 1 \mathrm{H}, \mathrm{J}=5.5,1.5 \mathrm{~Hz}) ; 7.79$ (td, $1 \mathrm{H}, \mathrm{J}=7.2,1.5 \mathrm{~Hz}) ; 7.75(\mathrm{td}, 1 \mathrm{H}, \mathrm{J}=5.5,2.0 \mathrm{~Hz}) ; 7.32-7.22$ $(\mathrm{m}, 8 \mathrm{H}) \mathrm{ppm}$; MS (70 eV): $358\left(\mathrm{M}^{+}, 21\right) ; 329$ (22); 105 (34); 78 (57); 77 (100). Anal. calcd. for $\mathrm{C}_{21} \mathrm{H}_{14} \mathrm{~N}_{2} \mathrm{O}_{2} \mathrm{~S}: \mathrm{C}, 70.37 ; \mathrm{H}, 3.93 ; \mathrm{N}, 7.81$. Found: C, 70.25; H, 4.10; N, 7.79.

Synthesis of betaines $7 \mathrm{a}, \mathrm{c}, \mathrm{e}, \mathrm{g}$. General procedure. To a suspension of the corresponding azinium salt ( $2 \mathrm{mmol})$ and $1.10 \mathrm{~g}(8 \mathrm{mmol})$ of $\mathrm{K}_{2} \mathrm{CO}_{3}$ in dry acetonitrile $(10 \mathrm{ml}), 0.24 \mathrm{ml}$ of phenyl isocyanate ( 2.2 $\mathrm{mmol}$ ) were added. The mixture was stirred for $20 \mathrm{~h}$ at room temperature. Then, the solid obtained was filtered off and purified by column chromatography (silica gel 60 A, 230-400 mesh; acetone for 7a or ethyl acetate for $\mathbf{7 c}, \mathbf{e})$.

1-Oxo-2-phenylpyrido[2,1-f][1,2,4]triazinium-3-olate (7a). $0.35 \mathrm{~g}(70 \%)$ of a white crystalline solid. Mp 308-309 ${ }^{\circ} \mathrm{C}\left(\mathrm{CH}_{3} \mathrm{CN}\right)$; IR (KBr): 1700, 1634, 1444, $1180 \mathrm{~cm}-1$; ${ }^{1} \mathrm{H}$ NMR (DMSO-d $)$ 8 8.71-8.67 (m,1 H); 8.33-8.29 (m, 1H); 7.98-7.93 (m, 2H); 7.50-7.24 (m, 5H) ppm; ${ }^{13} \mathrm{C}$ NMR (DMSO-d 6 ) $\delta 157.1 ; 153.2 ; 135.8$; $135.4 ; 133.8 ; 132.9 ; 129.6 ; 128.7 ; 128.4 ; 128.0 ; 125.7$ ppm; MS (70 eV) m/e (rel intensity): $239\left(\mathrm{M}^{+}, 12\right)$; 197 (29); 120 (11); 78 (100). Anal. calcd. for $\mathrm{C}_{13} \mathrm{H}_{9} \mathrm{~N}_{3} \mathrm{O}_{2}$ : C, 65.27; H, 3.79; $\mathrm{N}, 17.56$. Found: $\mathrm{C}, 65.05 ; \mathrm{H}$, $3.87 ; \mathrm{N}, 17.37$.

4-Oxo-3-phenyltriazinol 1,6-a]quinolinium-2-olate (7c). $0.38 \mathrm{~g}(66 \%)$ as a yellow solid. $\mathrm{Mp} 334-335^{\circ} \mathrm{C}$ $\left(\mathrm{CH}_{2} \mathrm{Cl}_{2} / \mathrm{EtOH}\right)$; IR (KBr) $1701,1630,1412,1193,1138 \mathrm{~cm}^{-1} ;{ }^{1} \mathrm{H}$ NMR (DMSO-d 6$) \delta 9.05$ (d, $1 \mathrm{H}, \mathrm{J}=8.8$ $\mathrm{Hz}) ; 8.46(\mathrm{~d}, 1 \mathrm{H}, \mathrm{J}=8.8 \mathrm{~Hz}) ; 8.28(\mathrm{dd}, 1 \mathrm{H}, \mathrm{J}=7.8,1.2 \mathrm{~Hz}) ; 8.25(\mathrm{~d}, 1 \mathrm{H}, \mathrm{J}=8.8 \mathrm{~Hz}) ; 8.09(\mathrm{ddd}, 1 \mathrm{H}, \mathrm{J}=$ $8.8,7.2,1.5 \mathrm{~Hz}) ; 8.02-7.95(\mathrm{~m}, 1 \mathrm{H}) ; 7.55-7.30(\mathrm{~m}, 5 \mathrm{H}) \mathrm{ppm}$; MS $(70 \mathrm{eV}) \mathrm{m} / \mathrm{e}$ (rel intensity): $289\left(\mathrm{M}^{+}, 36\right)$; 247 (61); 128 (100); 114 (65). Anal. calcd. for $\mathrm{C}_{17} \mathrm{H}_{11} \mathrm{~N}_{3} \mathrm{O}_{2}$ : C, 70.58; H, 3.83; N, 14.52. Found: C, 70.19; $\mathrm{H}, 4.15 ; \mathrm{N}, 14.19$.

1-Oxo-2-phenyltriazinol6,1-a]isoquinolinium-3-olate (7e). $0.43 \mathrm{~g}(74 \%)$ as yellow needles. Mp 330- 
$331^{\circ} \mathrm{C}\left(\mathrm{CH}_{2} \mathrm{Cl}_{2} / \mathrm{EtOH}\right) ; \mathrm{IR}(\mathrm{KBr}): 1689,1631,1457,1336,1138 \mathrm{~cm}^{-1} ;{ }^{1} \mathrm{H}$ NMR (DMSO-d 6 ) $\delta$ 9.67-9.63 (m, 1H); 8.53 (d, 1H, J = 7.3 Hz); 8.38 (d, 1H, J = 7.3 Hz); 8.19-8.14 (m, 1H); 7.92-7.86 (m, 2H); 7.53-7.29 (m, 5H); MS (70 eV) m/e (rel. intensity): $289\left(\mathrm{M}^{+}, 7\right) ; 247$ (38); 142 (14); 128 (100); 114 (48). Anal. calcd. for $\mathrm{C}_{17} \mathrm{H}_{11} \mathrm{~N}_{3} \mathrm{O}_{2}$ : C, 70.58; H, 3.83; N, 14.52. Found: C, 70.29, H, 3.91; N, 14.81 .

1-Oxo-2-phenyltriazino[1,6-b]isoquinolinium-3-olate $(7 \mathrm{~g})$. The final precipitate was washed with distilled water until neutral, and dried, yielding $7 \mathrm{~g}(0.34 \mathrm{~g}, 60 \%)$ as a yellow solid. $\mathrm{Mp}>350^{\circ} \mathrm{C}$ (DMF/EtOH); IR (KBr): $1692,1629,1485,1269,1210 \mathrm{~cm}^{-1} ;{ }^{1} \mathrm{H}$ NMR $\left(\mathrm{CF}_{3} \mathrm{COOD}\right) \delta 9.36(\mathrm{~s}, 1 \mathrm{H}) ; 8.98(\mathrm{~s}, 1 \mathrm{H}) ; 8.08(\mathrm{dd}, 1 \mathrm{H}$, $\mathrm{J}=8.2,1.2 \mathrm{~Hz}) ; 8.04(\mathrm{~d}, 1 \mathrm{H}, \mathrm{J}=7.8 \mathrm{~Hz}) ; 7.94-7.76(\mathrm{~m}, 2 \mathrm{H}) ; 7.16-6.88(\mathrm{~m}, 5 \mathrm{H}) ; \mathrm{MS}(70 \mathrm{eV}) \mathrm{m} / \mathrm{e}(\mathrm{rel}$ intensity): 289 ( $\left.\mathrm{M}^{+}, 11\right) ; 247$ (16); 142 (38); 128 (100). Anal.calcd. for $\mathrm{C}_{17} \mathrm{H}_{11} \mathrm{~N}_{3} \mathrm{O}_{2}$ : C, 70.58; H, 3.83; N, 14.52. Found: C, $70.31 ; \mathrm{H}, 4.04 ; \mathrm{N}, 14.70$.

Synthesis of betaines $\mathbf{7 b}, \mathbf{f}, \mathbf{h}, \mathbf{d}$. General procedure. To a stirred suspension of the corresponding azinium salt $(2 \mathrm{mmol})$ and $0.55 \mathrm{~g}(4 \mathrm{mmol})$ of $\mathrm{K}_{2} \mathrm{CO}_{3}$ in dichloromethane, $0.26 \mathrm{ml}(2,2 \mathrm{mmol})$ of phenyl isothiocyanate were added. The mixture was stirred for $20 \mathrm{~h}$ at room temperature. Then, the precipitate was isolated by filtration, washed with distilled water until neutral, and petroleum ether and finally recrystallized.

1-Oxo-2-phenylpyrido[2,1-f][1,2,4]triazinium-3-thiolate (7b). Recrystallization from $\mathrm{CH}_{2} \mathrm{Cl}_{2} / \mathrm{EtOH}$ yield $0.36 \mathrm{~g}$ of $7 \mathrm{~b}$ as a pale orange crystalline solid $(70 \%) . \mathrm{Mp} 212-213^{\circ} \mathrm{C}$. IR $(\mathrm{KBr}) 1701,1498,1417,1212 \mathrm{~cm}^{-1}$; ${ }^{1} \mathrm{H}$ NMR $(300 \mathrm{MHz}$, DMSO-d 6 ) $\delta 8.87(\mathrm{dd}, 1 \mathrm{H}, \mathrm{J}=6.1,1.2 \mathrm{~Hz}), 8.35(\mathrm{dd}, 1 \mathrm{H}, \mathrm{J}=7.9,1.9 \mathrm{~Hz}), 8.14$ (td, $1 \mathrm{H}, \mathrm{J}=7.8,1.2 \mathrm{~Hz}), 8.07$ (ddd, $1 \mathrm{H}, \mathrm{J}=7.8,6.1,1.9 \mathrm{~Hz}), 7.49-7.18(\mathrm{~m}, 5 \mathrm{H}) \mathrm{ppm} ;{ }^{13} \mathrm{C} \mathrm{NMR}(75.429$ MHz,DMSO-d 6 ) $\delta 176.8,154.8,138.9,136.4,136.3,136.0,130.2,130.0,128.9,128.8,125.7$ ppm; MS (70 eV) m/e (rel intensity) $255\left(\mathrm{M}^{+}, 11\right), 223$ (17), 106 (93), 78 (100). Anal. calcd. for $\mathrm{C}_{13} \mathrm{H}_{9} \mathrm{~N}_{3} \mathrm{OS}: \mathrm{C}, 61.16$; H, 3.55; N, 16.46. Found: C, 60.98; H 3.66; N, 16.71.

1-Oxo-2-phenyltriazino-[6,1-a]isoquinolinium-3-thiolate (7f). Work up of the mixture gave $0.37 \mathrm{~g}$ of $7 \mathrm{f}$ as an orange solid (61\%). Mp 258-259 (DMF). IR (KBr) 1679, 1465, 1370, $1232^{-1} ;{ }^{1} \mathrm{H}$ NMR $(300$ $\left.\mathrm{MHz}, \mathrm{CF}_{3} \mathrm{COOD}\right) \delta 9.98(\mathrm{~d}, 1 \mathrm{H}, \mathrm{J}=8.8 \mathrm{~Hz}), 8.84(\mathrm{~d}, 1 \mathrm{H}, \mathrm{J}=7.1 \mathrm{~Hz}), 8.70(\mathrm{~d}, 1 \mathrm{H}, \mathrm{J}=7.1 \mathrm{~Hz}), 8.40-8.21$ (m, 3H), 7.81-7.49 (m, 5H); MS (70 eV) m/e (rel intensity) $305\left(\mathrm{M}^{+}, 6\right), 273$ (17), 156 (12), 128 (100). Anal. calcd. for $\mathrm{C}_{17} \mathrm{H}_{11} \mathrm{~N}_{3} \mathrm{OS}$ : C, 66.87; H, 3.63; N, 13.76. Found: C,66.40; H, 3.96; N, 13.81 .

1-Oxo-2-phenyltriazino[1,6-b]isoquinolinium-3-thiolate (7h). $0.26 \mathrm{~g} \mathrm{(45 \% )}$ as a yellow solid. Mp 240 $241^{\circ} \mathrm{C}$ (DMF). IR (KR) $1681,1487,1450,1217 \mathrm{~cm}^{-1} ;{ }^{1} \mathrm{H}$ NMR $\left(300 \mathrm{MHz}, \mathrm{CF}_{3} \mathrm{COOD}\right) \delta 9.85(\mathrm{~s}, 1 \mathrm{H}), 9.50$ $(\mathrm{s}, 1 \mathrm{H}), 8.65(\mathrm{~d}, 1 \mathrm{H}, \mathrm{J}=8.5 \mathrm{~Hz}), 8.56(\mathrm{~d}, 1 \mathrm{H}, \mathrm{J}=8.3 \mathrm{~Hz}), 8.44(\mathrm{td}, 1 \mathrm{H}, \mathrm{J}=8.3,7.1,1.2 \mathrm{~Hz}), 8.35$ (ddd, $1 \mathrm{H}$, $\mathrm{J}=8.5,7.1,1.2 \mathrm{~Hz}), 7.82-7.50(\mathrm{~m}, 5 \mathrm{H}) \mathrm{ppm}$; MS $(70 \mathrm{eV}) \mathrm{m} / \mathrm{e}$ (rel intensity) $305\left(\mathrm{M}^{+}, 5\right), 273(8), 156(34)$, 128 (100). Anal. calcd. for $\mathrm{C}_{17} \mathrm{H}_{11} \mathrm{~N}_{3} \mathrm{OS}$ : C, 66.87; H, 3.63; N, 13.76. Found: $\mathrm{C}, 66.80 ; \mathrm{H}, 3.82 ; \mathrm{N}, 13.83$.

4-Oxo-3-phenyltriazino[1,6-a]quinolinium-2-thiolate (7d). After stirring at room temperature for $20 \mathrm{~h}$, by addition of distilled water $(20 \mathrm{ml})$ and extraction with dichloromethane $(3 \times 20 \mathrm{ml})$, the residue was treated with petroleum ether and the resulting precipitate was filtered off, giving $0.32 \mathrm{~g}$ of $\mathbf{7 d}$ as a red crystalline solid (53\%). Mp 213-214 ${ }^{\circ} \mathrm{C}\left(\mathrm{CH}_{3} \mathrm{CN}\right)$. IR (KBr) $1678,1468,1411,1205,1121 \mathrm{~cm}^{-1}$; ${ }^{1} \mathrm{H}$ NMR $(300 \mathrm{MHz}$,DMSO$\left.\mathrm{d}_{6}\right) \delta 9.06(\mathrm{~d}, 1 \mathrm{H}, \mathrm{J}=8.8 \mathrm{~Hz}), 8.67(\mathrm{~d}, 1 \mathrm{H}, \mathrm{J}=8.5 \mathrm{~Hz}), 8.35(\mathrm{dd}, 1 \mathrm{H}, \mathrm{J}=8.2,1.5 \mathrm{~Hz}), 8.29(\mathrm{~d}, 1 \mathrm{H}, \mathrm{J}=8.5$ $\mathrm{Hz}$ ), 8.17 (ddd, $1 \mathrm{H}, \mathrm{J}=8.8,7.2,1.5 \mathrm{~Hz}), 8.06-8.00(\mathrm{~m}, 1 \mathrm{H}), 7.53-7.37(\mathrm{~m}, 5 \mathrm{H}) \mathrm{ppm}$; MS $(70 \mathrm{eV}) \mathrm{m} / \mathrm{e}(\mathrm{rel}$ intensity) $305\left(\mathrm{M}^{+}, 22\right), 273(13), 156(11), 128(100)$. Anal. calcd. for $\mathrm{C}_{17} \mathrm{H}_{11} \mathrm{~N}_{3} \mathrm{OS}$ : C, 66.87; H, 3.63; N, 13.76. Found: $\mathrm{C}, 66.74 ; \mathrm{H}, 3.80 ; \mathrm{N}, 14.01$.

2-Phenyl-1-thiopyrido[2,1-f][1,2,4]triazinium-3-olate (9). To a suspension of $7 \mathbf{a}(0.48 \mathrm{~g}, 2 \mathrm{mmol})$ in dry pyridine $(10 \mathrm{ml})$, phosphorus pentasulfide $0.67 \mathrm{~g}(1.5 \mathrm{mmol})$ was added. After refluxing for $48 \mathrm{~h}$., distilled water $(20 \mathrm{ml})$ was added and the suspension was extracted with dichloromethane $(3 \times 50 \mathrm{ml})$, the organic phase was separated, dried with magnesium sulphate and concentrated to dryness. The residue was purified by 
column chromatography (silica 60 Merck, $230-400$ mesh), using dichloromethane/acetone (8:2), giving 0.26 $\mathrm{g}$ of 9 as an orange crystalline solid (50\%). Mp 308-309 ${ }^{\circ} \mathrm{C}$ (Acetone). IR (KBr) 1655, 1436, 1302, 1280, $1204,1167 \mathrm{~cm}^{-1}$; ${ }^{1} \mathrm{H}$ NMR $(300 \mathrm{MHz}$,DMSO-d 6 ) $\delta 8.83-8.77(\mathrm{~m}, 1 \mathrm{H}), 8.68-8.64(\mathrm{~m}, 1 \mathrm{H}), 8.00-7.93(\mathrm{~m}, 2 \mathrm{H})$, 7.53-7.19 (m, 5H) ppm; ${ }^{13} \mathrm{C}$ NMR $(75.429 \mathrm{MHz}) \delta 183.63,151.49,140.65,137.00,136.43,133.43,130.03$, 129.43, 129.30, 128.18, $127.74 \mathrm{ppm}$; MS $(70 \mathrm{eV}) \mathrm{m} / \mathrm{e}$ (rel intensity) $255\left(\mathrm{M}^{+}, 62\right), 213(100), 181(8), 78$ (17). Anal. calcd. for $\mathrm{C}_{13} \mathrm{H}_{9} \mathrm{~N}_{3} \mathrm{OS}$ : C, 61.16; H, 3.55; N, 16.46. Found: C, 60.91; H, 3.80; N, 16.21.

2-Phenyl-1-thiopyrido[2,1-f][1,2,4]triazinium-3-thiolate (10). Method A. A suspension of $7 \mathrm{a}(0.48 \mathrm{~g}$, $2 \mathrm{mmol})$ and $0.81 \mathrm{~g}(2 \mathrm{mmol})$ of Lawesson's reagent in dry toluene $(20 \mathrm{ml})$ were refluxed over $72 \mathrm{~h}$. After this time a mixture of two compounds was observed by t.l.c. Chromatography of the mixture (silica gel 60 Merck, $230-400$ mesh) with ethyl acetate gave $0.22 \mathrm{~g}$ of the betaine $10(40 \%)$ and $0.11 \mathrm{~g}$ of $\mathbf{7 b}(20 \%)$.

Method B. A suspension of $0.51 \mathrm{~g}(2 \mathrm{mmol})$ of the betaine 9 and $0.81 \mathrm{~g}(2 \mathrm{mmol})$ of Lawesson's reagent in dry toluene $(20 \mathrm{ml})$ were refluxed over $48 \mathrm{~h}$. After this time, the reaction mixture was concentrated to dryness and the residue purified by column chromatography (silica gel 60 Merck, 230-400 mesh), using dichloromethane/acetone (9:1) as eluents, giving $0.32 \mathrm{~g}$ of $\mathbf{1 0}$ as a brown-reddish solid $(63 \%) . \mathrm{Mp} 215-216^{\circ} \mathrm{C}$ (Acetone). IR (KBr) 1414, 1267, 1153, $1124 \mathrm{~cm}^{-1} ;{ }^{1} \mathrm{H}$ NMR (300 MHz,DMSO-d 6 ) $\delta 8.81$ (dd, $1 \mathrm{H}, \mathrm{J}=5.6$, $1.3 \mathrm{~Hz}$ ), 8.74 (dd, $1 \mathrm{H}, \mathrm{J}=7.9,2.2 \mathrm{~Hz}), 8.16-8.04(\mathrm{~m}, 2 \mathrm{H}), 7.50-7.11(\mathrm{~m}, 5 \mathrm{H}) ;$ MS $(70 \mathrm{eV}) \mathrm{m} / \mathrm{e}$ (rel intensity) $271\left(\mathrm{M}^{+}, 13\right), 213(32), 181$ (37), 78 (100). Anal. calcd. for $\mathrm{C}_{13} \mathrm{H}_{9} \mathrm{~N}_{3} \mathrm{~S}_{2}: \mathrm{C}, 57.54 ; \mathrm{H}, 3.34 ; \mathrm{N}, 15.48$. Found: C, $57.84 ; \mathrm{H}, 3.62 ; \mathrm{N}, 15.40$.

Synthesis of compounds 8 and 11. General procedure. To a suspension of the corresponding betaine $7 \mathbf{b} / 10$ (1 mmol) in ethyl acetate $(5 \mathrm{ml}), 0.44 \mathrm{ml}(4 \mathrm{mmol})$ of methyl iodide were added. After stirring at room temperature for $2 \mathrm{~h}$, the resulting precipitate was filtered off and washed with ethyl acetate. Recrystallization from ethanol gave $0.34 \mathrm{~g}(85 \%)$ of $\mathbf{8}$ and $0.33 \mathrm{~g}(81 \%)$ of $\mathbf{1 1}$ as yellow and orange crystalline solids respectively.

3-Methylthio-1-oxo-2-phenylpyrido[2,1-f] $[1,2,4]$ triazinium iodide (8). $\mathrm{Mp} 213-214^{\circ} \mathrm{C}(\mathrm{EtOH}) . \mathrm{IR}(\mathrm{KBr})$ $1727,1635,1543,1443,1271 \mathrm{~cm}^{-1},{ }^{1} \mathrm{H}$ NMR $(300 \mathrm{MHz}$, DMSO-d 6$) \delta 9.44(\mathrm{~d}, 1 \mathrm{H}, \mathrm{J}=6.3 \mathrm{~Hz}), 8.78(\mathrm{dd}, 1 \mathrm{H}$, $\mathrm{J}=7.8,1.7 \mathrm{~Hz}), 8.70(\mathrm{t}, 1 \mathrm{H}, \mathrm{J}=7.8 \mathrm{~Hz}), 8.53-8.46(\mathrm{~m}, 1 \mathrm{H}), 7.69-7.44(\mathrm{~m}, 5 \mathrm{H}), 2.61(\mathrm{~s}, 3 \mathrm{H}) \mathrm{ppm}$; MS $(70$ eV) $\mathrm{m} / \mathrm{e}$ (rel intensity) $255\left(\mathrm{M}^{+}-15,6\right), 223$ (17), 142 (58), 106 (67), 78 (100). Anal. calcd. for $\mathrm{C}_{14} \mathrm{H}_{12} \mathrm{~N}_{3}$ OSI: C, $42.33 ; \mathrm{H}, 3.05 ; \mathrm{N}, 10.58$. Found: $\mathrm{C}, 41.85 ; \mathrm{H}, 2.98 ; \mathrm{N}, 10.23$.

3-Methylthio-1-thio-2-phenylpyrido/2,1-f] $[1,2,4]$ triazinium iodide (11). $\mathrm{Mp} 225-226^{\circ} \mathrm{C}(\mathrm{EtOH}) . \mathrm{IR}(\mathrm{KBr})$ 1533, 1468, 1440, 1310, $1271 \mathrm{~cm}^{-1}$ : ${ }^{1} \mathrm{H}$ NMR (300 MHZ,DMSO-d 6 ) $\delta 9.36(\mathrm{~d}, 1 \mathrm{H}, \mathrm{J}=6.3 \mathrm{~Hz}$ ), 9.07 (dd, $1 \mathrm{H}, \mathrm{J}=8.1,1.3 \mathrm{~Hz}), 8.67(\mathrm{t}, 1 \mathrm{H}, \mathrm{J}=8.1,7.8 \mathrm{~Hz}), 8.53-8.47(\mathrm{~m}, 1 \mathrm{H}), 7.72-7.39(\mathrm{~m}, 5 \mathrm{H}), 2.60(\mathrm{~s}, 3 \mathrm{H}) \mathrm{ppm}$; MS $\left(70 \mathrm{eV}\right.$ ) m/e (rel intensity) $254\left(\mathrm{M}^{+}-32,31\right), 213$ (24), 181 (28), 78 (38). Anal. calcd. for $\mathrm{C}_{14} \mathrm{H}_{12} \mathrm{~N}_{3} \mathrm{~S}_{2} \mathrm{I}$ : C, $40.70 ; \mathrm{H}, 2.93 ; \mathrm{N}, 10.17$. Found: C, 41.00; H, 3.12; N, 10.22 .

\section{ACKNOWLEDGEMENTS}

We wish to express our thanks to the Comision Interministerial de Ciencia y Tecnologia (C.I.C.Y.T) for financial support (Project PB90-0284), and to the Ministerio de Educación y Ciencia by a grant to one of us (A.M.C).

\section{REFERENCES}

1. Bátori, S.; Juhász-Riedl, Zs.; Sandor, P.; Messmer, A. J. Heterocyclic Chem. 1986, 23, 375.

2. Bátori, S.; Messmer, A. J. Heterocyclic Chem. 1988, 25, 437. 
3. Hajós, G.; Messmer, A; Batori, S.; Riedl, Z. Bull. Soc. Belg.1992, 101, 597.

4. Bátori, S.; Messmer, A. J. Heterocyclic Chem. 1990, 27, 1673.

5. Kakehi, A.; Ito, S.; Uchiyama, K.; Konno, Y. Chem. Lett. 1976, 413.

6. Katritzky, A.R.; Ballesteros, P.; Tárraga, A. J. Chem. Soc. Perkin Trans.I. 1981, 1495.

7. Molina, P.; Tárraga, A.; Lorenzo Peña, M; Hurtado, E.; Vilaplana, M.J. Tetrahedron Lett. 1982, 23, 2985.

8. Molina, P.; Tárraga, A.; Vilaplana, M.J.; Hurtado, M.; Lorenzo, M. J. Chem. Soc. Perkin Trans.I. 1983, 1395.

9. Molina, P.; Tárraga, A.; Lorenzo-Peña, M.; Espinosa, A. Heterocycles.1987, 26, 2183.

10. Ollis, D.W.; Stanforth, S.P. Tetrahedron. 1985,41, 223.

11. Glover, E.E.; Loadman M.J.R. J. Chem. Soc. (C). 1967, 2391.

12. Tamura, Y.; Minamikawa, J.; Ikeda, M. Synthesis. 1977, 1. 
